University of Wollongong

Research Online

Faculty of Engineering - Papers (Archive)

Faculty of Engineering and Information

Sciences

July 2002

\title{
Photoinduced band-bending effect of low temperature GaAs on AIGaAs/ InGaAs/GaAs modulation-doped transistors
}

\author{
P. A. Folkes \\ G. Gumbs \\ City University of New York, USA
}

US Army Research Laboratory, Maryland, USA

W. Xu

University of Wollongong

Follow this and additional works at: https://ro.uow.edu.au/engpapers

Part of the Engineering Commons

https://ro.uow.edu.au/engpapers/172

\section{Recommended Citation}

Folkes, P. A.; Gumbs, G.; and Xu, W.: Photoinduced band-bending effect of low temperature GaAs on AlGaAs/InGaAs/GaAs modulation-doped transistors 2002.

https://ro.uow.edu.au/engpapers/172

Research Online is the open access institutional repository for the University of Wollongong. For further information contact the UOW Library: research-pubs@uow.edu.au 


\title{
Photoinduced band-bending effect of low temperature GaAs on AIGaAs/InGaAs/GaAs modulation-doped transistors
}

\author{
Patrick A. Folkes ${ }^{\text {a) }}$ \\ U.S. Army Research Laboratory, Adelphi, Maryland 20783-1197 \\ Godfrey Gumbs ${ }^{\text {b) }}$ \\ Department of Physics, Hunter College of the City University of New York, New York 10021 \\ Wen $\mathrm{Xu}$ \\ Institute of Superconductivity and Electronic Materials, University of Wollongong, Wollongong, NSW 2522, \\ Australia
}

(Received 4 February 2002; accepted for publication 11 April 2002)

\begin{abstract}
Low temperature photoluminescence (PL) measurements on pseudomorphic modulation-doped transistors (PHEMTs) with a low-temperature (LT) GaAs layer in the GaAs buffer layer clearly show a decrease in the quantum well PL transition energies compared to a PHEMT with no LT GaAs. Self-consistent calculations of the quantum well PL transition energies and oscillator strengths show that the observed decrease in PL energies can be attributed to a larger photoinduced band bending in PHEMTs with an undoped GaAs/LT GaAs interface compared to the photoinduced band bending in PHEMTs with an undoped GaAs/semi-insulating GaAs interface. (C) 2002 American Institute of Physics. [DOI: 10.1063/1.1483132]
\end{abstract}

The properties, ${ }^{1}$ device applications, ${ }^{2,3}$ and mechanism $^{4-8}$ of low temperature (LT) GaAs that is grown by molecular beam epitaxy (MBE) with a substrate temperature of around $200-300^{\circ} \mathrm{C}$ have been studied intensively. ${ }^{1-10}$ Low temperature photoluminescence (PL) has been used to characterize the two-dimensional electron gas (2DEG) in a pseudomorphic high electron mobility transistor (PHEMT). ${ }^{11}$ Recently, we published the results of LT PL measurements on a number of PHEMT heterostructures which have a LT GaAs layer embedded in the nominally undoped GaAs buffer layer at various depths below the quantum well $(\mathrm{QW}) .{ }^{12}$ Our results clearly showed a decrease in the QW PL transition energies (redshift) of the PHEMTs with a LT GaAs layer compared to structures with no LT GaAs. Calculations of the electron and hole subband energies, taking into account the Fermi level pinning at the undoped GaAs/LT GaAs/semiinsulating GaAs interfaces, the thickness of the undoped GaAs layer, and assuming negligible hole density in the undoped GaAs, qualitatively agree with the experimental results on the structures with a LT GaAs layer but cannot explain the observed higher PL energies of the structure with no LT GaAs layer. ${ }^{13}$ The preliminary calculations used to gain insight into the observed effects ${ }^{12}$ gave an inaccurate dependence of the conduction subband energy levels on substrate voltage and could not rule out the possibility that the observed redshift in PL energies can be attributed to photoinduced changes in the acceptor compensation in the undoped GaAs. In this communication we present theoretical results on the dependence of the PL transition energies and

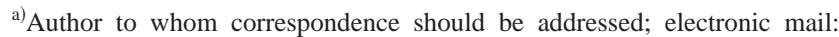
pfolkes@arl.army.mil

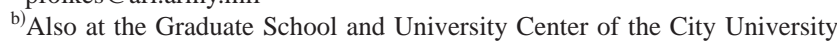
of New York, 365 Park Ave., New York, NY 10036.
}

oscillator strengths on the substrate voltage, which agree with our experimental results and confirm that the mechanism for the redshift in PL energies is the quantum confined Stark effect. ${ }^{14}$ Our results suggest that the observed redshift in PL energies can be attributed to photoinduced band bending in the undoped GaAs caused by the trapping of photoexcited holes at the undoped GaAs/LT GaAs interface. These results also show that the photoinduced band bending in PHEMTs with an undoped GaAs/LT GaAs interface is large compared to the photoinduced band bending in PHEMTs with an undoped GaAs/semi-insulating GaAs interface.

The PHEMTs used in this study were grown by MBE on semi-insulating GaAs. Characteristics of these heterostructures and experimental details have been previously reported. ${ }^{12}$ The observed $6 \mathrm{~K} \mathrm{PL}$ spectrum ${ }^{12}$ of the sample without the LT GaAs shows a $16 \mathrm{meV}$ wide (full width half maximum) asymmetric feature with a peak at $1255 \mathrm{meV}$ and a relatively sharp feature at $1315 \mathrm{meV}$ with a width of $3 \mathrm{meV}$ and a factor of approximately 12 greater peak intensity compared to the $1255 \mathrm{meV}$ peak. The observed difference in peak energies, the dependence of the PL on the QW-LT GaAs spacing, and theoretical modeling lead us to conclude that the observed PL can be attributed to the recombination of $n=1$ and $n=2$ subband electrons from the degenerate 2DEG with $m=1$ heavy holes in the InGaAs QW. The PL from the $n=1$ electron to $m=1$ heavy hole transition (designated $11 \mathrm{H}$ ) comes from the recombination of free electron-hole $(e-h)$ pairs. The relatively large peak intensity, symmetric line shape, and narrow width of the PL from the $n=2$ electron to $m=1$ heavy hole transition (designated $21 H$ ) suggest that this PL feature primarily comes from the recombination of $n=2$ subband excitons. The $6 \mathrm{~K}$ PL data from the structures with a LT GaAs layer show that both the $11 \mathrm{H}$ and the $21 \mathrm{H}$ transitions come from the recombination 
of free $e-h$ pairs. The structures with a LT GaAs layer exhibit a decrease in the $11 \mathrm{H}$ and $21 \mathrm{H}$ PL energies with increasing QW-LT GaAs spacing for spacings up to $1.6 \mu \mathrm{m} .{ }^{12}$ Compared to the structure without LT GaAs, the observed redshifts of the $11 \mathrm{H}$ and $21 \mathrm{H}$ PL energies, for the structure with QW-LT spacing of $1.6 \mu \mathrm{m}$, are 17.3 and $21.7 \mathrm{meV}$, respectively.

Self-consistent calculations have shown that the conduction subband energies in back-gated heterostructures ${ }^{15}$ are sensitive to the backgate-induced variations in the substrate potential far from the QW. This suggests that, under constant illumination, the LT GaAs layer in our heterostructures induces an increase in the band bending in the undoped GaAs compared to the structure with no LT GaAs, resulting in a decrease in the PL transition energies. During constant illumination $e-h$ pairs are created by the absorption of photons in the undoped GaAs layer. The strong modulation dopinginduced electric field in the undoped GaAs layer sweeps photoexcited electrons into the QW, while the photoexcited holes are driven toward the substrate. For the case of the PHEMT with no LT GaAs, the resultant accumulation of photoexcited holes at the undoped GaAs/semi-insulating GaAs interface generates a steady-state photoinduced voltage which causes band bending. ${ }^{16,17}$ For the case of our heterostructures with a LT GaAs layer, the photoexcited holes are trapped at the undoped GaAs/LT GaAs interface by the high density of defects in LT GaAs, resulting in a faster removal of holes from the undoped GaAs. In addition, the LT GaAs decreases the electron injection current from the 2DEG to the LT GaAs interface. These effects of the LT GaAs combine to increase the $e-h$ recombination time of the PHEMT, resulting in a larger steady-state photoinduced voltage. Our observations ${ }^{12}$ show that the photoinduced voltage saturates for laser intensities as small as $0.2 \mathrm{~W} / \mathrm{cm}^{2}$ ostensibly when the hole generation current to the interface equals the hole recombination current due to $e-h$ recombination at or near the undoped GaAs/LT GaAs interface.

Self-consistent Schrodinger-Poisson calculations of the conduction and valence band energy levels and wave functions for this heterostructure were carried out using a Coulomb interaction, which comprises both the Hartree and exchange-correlation terms. ${ }^{18}$ The coupling between electrons and heavy hole states is accounted for. The Hartree term is calculated from the Poisson equation and the exchange-correlation term from density functional theory. The valence band calculation takes into account the renormalized band gap of the strained InGaAs layer. ${ }^{19}$ It is assumed that the hydrostatic part of the strain acts only on the conduction band edge. This is a reasonable assumption since published data show that the conduction band deformation potential is at least ten times larger than the valence band deformation potential. The variation of the potential between the QW and undoped GaAs/LT GaAs interface is determined by band bending, resulting from the transfer of electrons from the AlGaAs layer to the InGaAs QW, the depletion of holes from the undoped GaAs, and the nonequilibrium photoinduced voltage. Since the hole concentration in the undoped GaAs is unknown we fixed the potential at a point $2000 \AA$ from the AlGaAs/InGaAs QW interface, designated
$V_{r}$, as a boundary condition in our calculations. From the Fermi energy pinning at the surface and the known concentration of the doped layers above the QW, we determine that the potential at the GaAs/AlGaAs interface $\approx 0.35 \mathrm{~V}$. We set the Fermi energy $E_{F}=0$. The calculations are repeated for various $V_{r}$ to model the band-bending effect of the photoinduced voltage.

Our calculations confirm that the $n=1$ and $n=2$ conduction subbands are occupied, and good agreement with the magnetotransport-determined electron densities is obtained if the effective AlGaAs doping is $4 \times 10^{18} \mathrm{~cm}^{-3}$. The calculations show that the $n=1$ heavy hole groundstate subband energy is insensitive to variations in $V_{r}$, which implies that the observed decrease in PL energies is attributable to a decrease in the conduction subband energies. Figure 1 shows the theoretical $n=1$ and $n=2$ conduction subband energies as a function of $V_{r}$. The figure confirms that the conduction subband and the PL transition energies are sensitive to $V_{r}$. From the SdH measurements of the 2DEG density we determine that the $n=2$ subband energy is approximately -2 $\mathrm{meV}$ for the structure with no LT GaAs. Figure 1 shows that this corresponds to $V_{r}=0.42 \mathrm{~V}$ for the structure with no LT GaAs. Since the heavy hole ground state energy (not shown in Fig. 1) is not sensitive to $V_{r}$, the observed maximum redshift of the $11 \mathrm{H}$ and $21 \mathrm{H} \mathrm{PL}$ energies, $17 \mathrm{meV}$ and 21 $\mathrm{meV}$, respectively, can be obtained if $V_{r}=0.12 \mathrm{~V}$; this is close to a flatband condition in the undoped GaAs layer. For the structure with the $1.6 \mu \mathrm{m}$ undoped GaAs layer, the photoinduced voltage at the undoped GaAs/LT GaAs interface, which reduces $V_{r}$ and flattens the bands, is expected to have a maximum value, $\Delta V \approx$ band gap $\sim 1.5 \mathrm{~V}$. Using a depletion capacitance model, the interface density of photoexcited holes $\Delta n$ can be estimated by $\Delta n \approx \epsilon \Delta V / e d$, where $\epsilon$ is the GaAs dielectric constant, $e$ is the electron charge, and $d$ is the width of the undoped GaAs layer. Using $\Delta V$ $=1.5 \mathrm{~V}$ and $d=1.6 \mu \mathrm{m}$, we obtain $\Delta n \approx 6 \times 10^{10} \mathrm{~cm}^{-2}$, which can be obtained with a laser intensity of $0.2 \mathrm{~W} / \mathrm{cm}^{2}$ if we assume an $e-h$ recombination lifetime $\sim 10^{-7}$ s. This is a reasonable assumption given the above effects of the LTGaAs on the PHEMT. This photoinduced band-bending model is consistent with the observed decrease in the transition PL energies as the QW-LT spacing increases, due to the increased absorption of photons in the undoped GaAs layer. The nonequilibrium photoinduced band-bending model is also consistent with the observed $n=2$ subband 2DEG densities in our structures.

The calculated subband energies are insensitive to variations in the acceptor concentration in the undoped GaAs at a fixed value of $V_{r}$. This rules out the possibility that the observed redshift in PL energies can be attributed to photoinduced changes in the acceptor compensation in the undoped GaAs. Excellent agreement between the observed and the calculated values of $11 \mathrm{H}$ and $21 \mathrm{H}$ energies are obtained using the published strained, ${ }^{19}$ renormalized ${ }^{20}$ band gap parameters. For example, using a value of $1.22 \mathrm{eV}$ for the strained InGaAs band gap and a band gap renormalization of $8 \mathrm{meV}$ with $V_{r}=0.42 \mathrm{~V}$, the calculated values of the $11 \mathrm{H}$ and $21 \mathrm{H}$ energies are 1.255 and $1.311 \mathrm{eV}$, respectively. The observed 


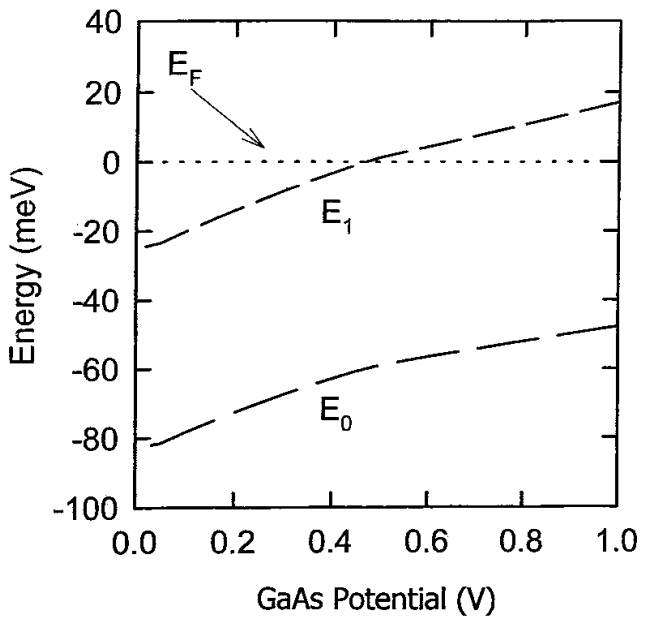

FIG. 1. Calculated QW conduction ground state $E_{0}$ and first excited state $E_{1}$ subband energies as a function of the undoped GaAs potential $V_{r}$ at a point $2000 \AA$ from the QW.

$11 H$ and $21 H$ peak energies are 1.255 and $1.315 \mathrm{eV}$, respectively.

Comparison of the corresponding ratios of QW peak PL intensity and the calculated transition oscillator strength (which is proportional to the integral of the square of the electron-hole wave function overlap), has been used to estimate the enhancement $\eta$ of PL near $E_{F}$ due to the manybody Fermi edge singularity. ${ }^{21}$ However, estimates of $\eta$ obtained in this manner could be misleading since PL broadening, which decreases the transition peak PL intensity, may differ significantly for $11 H$ and $21 H$ transitions. The observed PL intensity could also be affected by nonequilibrium effects on the joint density of states and the QW occupation probabilities. PL from free $e-h$ pairs is broadened by several mechanisms, including direct recombination of thermally distributed holes and electrons with the same wave vector, indirect recombination processes which are facilitated by the scattering of carriers in the QW by ionized impurities in the doped AlGaAs barrier, ${ }^{22}$ and QW inhomogeneities. Excitonic PL is primarily broadened by QW inhomogeneities. We calculated the $21 H$ to $11 H$ oscillator strength ratio $r$ for our $150 \AA$ InGaAs QW and compared $r$ with the corresponding experimental ratio of the integrated PL intensities of the $21 H$ and the $11 H$ features. The experimental integrated PL intensity ratio of the $21 H$ to $11 H$ features varies over the narrow range 3-3.4, and the calculated value of $r$ is $\sim 2.3$ over the range of $V_{r}$ relevant for our structures. These results confirm that our assignment of the observed QW transitions is correct, and suggest that we are observing a small enhancement of the PL near $E_{F}$. Since the $n=2$ subband is around $2 \mathrm{meV}$ below $E_{F}$, the ratio of the experimental integrated PL intensities of the $21 \mathrm{H}$ to the $11 \mathrm{H}$ features divided by $r$ gives an approximate value of $\eta$. For the structure with no LTGaAs, the $n=2$ subband exciton leads to an enhancement $\eta \approx 3 / 2.3=1.3$. In the case of the other structures the PL comes from free $e-h$ pairs; the observed PL enhancement $\eta \approx 3.4 / 2.3=1.5$ can be attributed to multiple $e-h$ scattering near $E_{F},{ }^{23}$ which leads to an increase in the $21 \mathrm{H}$ oscillator strength. Note that a comparison of $r$ with the ratio of the observed PL peak intensities in our structures would give an unrealistically large estimate of the enhancement of PL near $E_{F}$.

In summary, LT PL measurements on pseudomorphic modulation-doped transistors with a LT GaAs layer in the GaAs buffer layer clearly show a decrease in the QW PL transition energies compared to a structure with no LT GaAs. Self-consistent calculations of the PL transition energies and oscillator strengths verify that we have observed the $11 \mathrm{H}$ and $21 \mathrm{H}$ transitions. Our calculations suggest that the observed decrease in PL energies can be attributed to a larger photoinduced band bending in PHEMTs with an undoped GaAs/LT GaAs interface compared to the photoinduced band bending in PHEMTs with an undoped GaAs/semi-insulating GaAs interface. The photoinduced band-bending effect of LT GaAs may be relevant to the understanding of PL measurements on heterostructures which have a LT GaAs layer and the design of high speed photodetectors and photovoltaic devices.

The authors gratefully acknowledge discussions with H.-L. Cui. G.G. acknowledges the partial support of Grant No. 4137308-02 from NIH.

${ }^{1}$ F. W. Smith, A. R. Calawa, C-L Chen, M. J. Manfra, and L. J. Mahoney, IEEE Electron Device Lett. 9, 77 (1988).

${ }^{2}$ P. M. Solomon, S. L. Wright, and F. J. Canora, IEEE Electron Device Lett. 12, 117 (1991).

${ }^{3}$ B. J-F Lin, C. P. Kocot, D. E. Mars, and R. Jaeger, IEEE Trans. Electron Devices 37, 46 (1990).

${ }^{4}$ M. Kaminska, Z. Liliental-Weber, E. R. Weber, T. George, J. B. Kortright, F. W. Smith, B-Y. Tsaur, and A. R. Calawa, Appl. Phys. Lett. 54, 1881 (1989).

${ }^{5}$ M. O. Manasreh, D. C. Look, K. R. Evans, and C. E. Stutz, Phys. Rev. B 41, 10272 (1990).

${ }^{6}$ D. C. Look, D. C. Walters, M. O. Manasreh, J. R. Sizelove, C. E. Stutz, and K. R. Evans, Phys. Rev. B 42, 3578 (1990).

${ }^{7}$ X. Liu, A. Prasad, W. M. Chen, A. Kurpiewski, A. Stoschek, Z. Liliental-Weber, and E. R. Weber, Appl. Phys. Lett. 65, 3002 (1994).

${ }^{8}$ A. C. Warren, J. M. Woodall, J. L. Freeouf, D. Grishchkowsky, D. T. McInturff, M. R. Melloch, and N. Otsuka, Appl. Phys. Lett. 57, 1331 (1990).

${ }^{9}$ F. W. Smith et al., Appl. Phys. Lett. 54, 890 (1989).

${ }^{10}$ S. Gupta, M. Y. Frankel, J. A. Valdmanis, J. F. Whitaker, G. A. Mourou, F. W. Smith, and A. R. Calawa, Appl. Phys. Lett. 59, 3276 (1991).

${ }^{11}$ C. Colvard, N. Nouri, H. Lee, and D. Ackley, Phys. Rev. B 39, 8033 (1989).

${ }^{12}$ P. A. Folkes, D. Smith, R. A. Lux, W. Zhou, R. Thompson, R. Moerkirk, M. Lemeune, P. Cooke, and K. Brown, Appl. Phys. Lett. 69, 2234 (1996).

${ }^{13}$ W. D. Sun, F. H. Pollak, P. A. Folkes, and G. Gumbs, J. Electron. Mater. 28, L38 (1999).

${ }^{14}$ D. A. B. Miller, D. S. Chemla, T. C. Damen, A. C. Gossard, W. Wiegmann, T. H. Wood, and C. A. Burrus, Phys. Rev. B 32, 1043 (1985).

${ }^{15}$ B. Vinter, Solid State Commun. 48, 151 (1983).

${ }^{16}$ A. Kastalsky and J. C. M. Hwang, Solid State Commun. 51, 317 (1984).

${ }^{17}$ F. Stern, Surf. Sci. 174, 425 (1986).

${ }^{18}$ F. Stern and S. Das Sarma, Phys. Rev. B 30, 840 (1984).

${ }^{19}$ J.-Y. Marzin, M. N. Charasse, and B. Sermage, Phys. Rev. B 31, 8298 (1985).

${ }^{20}$ D. A. Kleinman and R. C. Miller, Phys. Rev. B 32, 2266 (1985).

${ }^{21}$ M. S. Skolnick, D. M. Whittaker, P. E. Simmonds, T. A. Fisher, M. K. Saker, J. M. Rorison, R. S. Smith, P. B. Kirby, and C. R. White, Phys. Rev. B 43, 7354 (1991).

${ }^{22}$ S. K. Lyo and E. D. Jones, Phys. Rev. B 38, 4113 (1988).

${ }^{23}$ G. D. Mahan, Phys. Rev. 153, 882 (1967). 